\begin{tabular}{|c|c|c|}
\hline \multirow{3}{*}{$\begin{array}{r}\text { Case Reports in } \\
\text { Gastroenterology }\end{array}$} & \multirow{2}{*}{\multicolumn{2}{|c|}{ Case Rep Gastroenterol 2013;7:202-207 }} \\
\hline & & \\
\hline & $\begin{array}{l}\text { DOI: } 10.1159 / 000351170 \\
\text { Published online: IVlay 4, } 2013\end{array}$ & $\begin{array}{l}\text { ( ) } 2013 \text { S. Karger AG, Basel } \\
1662-0631 / 13 / 0072-0202 \$ 38.00 / 0 \\
\text { www.karger.com/crg }\end{array}$ \\
\hline
\end{tabular}

\title{
Mirizzi Syndrome with Endoscopic Ultrasound Image
}

\author{
K. Rayapudi P. Gholami M. Olyaee \\ Kansas University Medical Center, Kansas City, Kans., USA
}

\section{Key Words}

Mirizzi syndrome · Gallstone · Endoscopic ultrasound · Obstructive jaundice

\begin{abstract}
We describe a 66-year-old Caucasian man with type 1 Mirizzi syndrome diagnosed on endoscopic ultrasound. He presented with acute onset of jaundice, malaise, dark urine over 3-4 days, and was found to have obstructive jaundice on lab testing. CT scan of the abdomen showed intrahepatic biliary ductal dilation, a $1.5 \mathrm{~cm}$ common bile duct (CBD) above the pancreas, and possible stones in the CBD, but no masses. Endoscopic retrograde cholangiopancreatography (ERCP) by a community gastroenterologist failed to cannulate the CBD. At the University Center, type 1 Mirizzi syndrome was noted on endoscopic ultrasound with narrowing of the CBD with extrinsic compression from cystic duct stone. During repeat ERCP, the $C B D$ could be cannulated over the pancreatic duct wire. A mid CBD narrowing, distal CBD stones, proximal $C B D$ and extrahepatic duct dilation were noted, and biliary sphincterotomy was performed. A small stone in the distal $C B D$ was removed with an extraction balloon. The cystic duct stone was moved with the biliary balloon into the $C B D$, mechanical basket lithotripsy was performed and stone fragments were delivered out with an extraction balloon. The patient was seen 7 weeks later in the clinic. Skin and scleral icterus had cleared up and he is scheduled for an elective cholecystectomy. Mirizzi syndrome refers to biliary obstruction resulting from impacted stone in the cystic duct or neck of the gallbladder and commonly presents with obstructive jaundice. Type 1 does not have cholecystocholedochal fistulas, but they present in types 2, 3 and 4. Surgery is the mainstay of therapy. Endoscopic treatment is effective and can also be used as a temporizing measure or definitive treatment in poor surgical risk candidates.
\end{abstract}


Rayapudi et al.: Mirizzi Syndrome with Endoscopic Ultrasound Image

\section{Introduction}

Pablo Luis Mirizzi, an Argentine surgeon, first described the syndrome in 1948 [1]: biliary obstruction resulting from impacted stone in the cystic duct or the gallbladder neck. It commonly presents with obstructive jaundice, with no definite unique features. Anatomical variations with long cystic duct parallel to the common hepatic duct or a low insertion of the cystic duct predispose to development of this syndrome. It has been noted to occur in up to $5.7 \%$ of all cholecystectomies, and $89.5 \%$ of patients with cholecystoenteric fistula had an associated Mirizzi syndrome in a retrospective analysis [2]. Four types of Mirizzi syndrome have been described which are detailed later in this article.

Mirizzi syndrome needs to be considered in the differential diagnosis of any patient presenting with obstructive jaundice. Right upper quadrant pain, jaundice and fever are common presenting symptoms [3]. Ultrasound and CT scan are common imaging modalities in patients presenting with obstructive jaundice. Ultrasound may reveal presence of gallstones or a contracted gallbladder. CT scan does not add to sonographic findings with respect to imaging of the stone and biliary obstruction; however it can help detect malignancy if enlarged porta hepatis lymph nodes or hepatic metastases are noted [4]. When suspected, preoperative cholangiography can be used to determine the presence or absence of a fistula [4]. Preoperative diagnosis is difficult to make in many cases. Only 10 out of 25 patients with this condition who were studied by Zhong and Gong [5] were diagnosed preoperatively. Magnetic resonance cholangiopancreatography (MRCP), endoscopic retrograde cholangiopancreatography (ERCP) and percutaneous transhepatic cholangiography (PTC) are the options for preoperative cholangiography. Therapeutic intervention would be possible during ERCP or PTC, but not during MRCP. ERCP can also be advantageous since visualization of the extrahepatic bile ducts is superior to PTC. Ampullary sphincterotomy with removal of bile duct stones is possible during ERCP. Surgery is the mainstay of therapy for this syndrome. However, endoscopic treatment of Mirizzi syndrome has been shown to be effective [6]. Endoscopic treatment with or without lithotripsy can also be used as a temporizing measure prior to surgery or as a definitive treatment in poor surgical risk candidates. Electrohydraulic lithotripsy [7] along with sphincterotomy, mechanical lithotripsy, dissolution therapy with methyl tert-butyl ether, extracorporeal shockwave lithotripsy and common bile duct (CBD) stents [8] have been utilized during endoscopic treatment. Open cholecystectomy is the major modality of management although a laparoscopic approach may be justified in some patients with type 1 variant of this syndrome $[9,10]$. Surgery for Mirizzi syndrome constitutes a laparoscopic challenge and conversion to open surgery has been recommended if local anatomy is not clear [11]. Association of Mirizzi syndrome with gallbladder cancer has also been demonstrated [12].

Herein, we describe a 66-year-old man who presented with obstructive jaundice and was diagnosed with Mirizzi syndrome type 1 on endoscopic ultrasound (EUS) exam. An EUS image is presented in this report (fig. 1). EUS images depicting this syndrome are rare. To our knowledge, there is only one other case report [1] with published an EUS image of Mirizzi syndrome.

\section{Case Report}

A 66-year-old man with a history of severe peripheral vascular disease and left iliac artery stent placement was seen in his primary care doctor's clinic for acute onset of jaundice, malaise and dark urine over 3-4 days. He had a dull, aching epigastric and right upper 
abdominal pain for 2 weeks prior to presentation. He could not relate worsening pain to meals and could not identify any inciting factors for the pain. Pain lasted from a few minutes to an hour once it occurred and it happened approximately 3-4 times daily. He had no history of nausea or vomiting. Jaundice developed rapidly in the 3-4 days prior to presentation, which made him visit his primary care doctor. He had been feeling tired most of the day for the past 2-3 weeks. Physical exam demonstrated scleral and skin icterus and minimal epigastric tenderness on deep palpation. Laboratory testing showed elevated liver enzymes: AST 92 U/l (normal 7-40 U/l), ALT 145 U/l (normal 7-56 U/l), ALP 443 U/l (normal 25$110 \mathrm{U} / \mathrm{l}$ ) and total bilirubin $25.3 \mathrm{mg} / \mathrm{dl}$ (normal $0.3-1.2 \mathrm{mg} / \mathrm{dl}$ ), direct bilirubin $18.9 \mathrm{mg} / \mathrm{dl}$. Complete blood count was normal. CT scan of the abdomen revealed intrahepatic biliary ductal dilation, a $1.5 \mathrm{~cm}$ CBD above the pancreas and rounded radiopaque densities (possibly stones ranging from 3 to $4 \mathrm{~mm}$ ) in the CBD within the pancreas. The pancreatic duct was normal. No masses or enlarged lymph nodes were noted. His primary care provider referred him to a community gastroenterologist who performed ERCP. During ERCP, the CBD could not be cannulated or opacified and the pancreatic duct was normal. The patient was referred to the University Gastroenterology Center for repeat ERCP. He arrived 2 days later for a repeat attempt. Labs were repeated prior to the procedure and showed AST

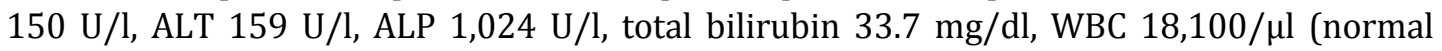
$4,500-11,000 / \mu \mathrm{l}$ ) and INR 1.3 (range 0.8-1.2). EUS was performed prior to ERCP to evaluate the bile ducts and pancreas further and to determine the cause of biliary obstruction. Type 1 Mirizzi syndrome was noted on EUS (fig. 1), showing proximal CBD dilation, narrowing of the CBD due to extrinsic compression from cystic duct stone, acoustic shadow from cystic duct stone, stone in the cystic duct and distal CBD stone. Repeat ERCP was performed and the CBD was cannulated over the pancreatic duct wire. A mid CBD narrowing, distal CBD stones, proximal CBD and extrahepatic duct dilation were noted, and biliary sphincterotomy was performed. An extraction balloon was used to remove the small stone from the distal CBD. Cholangiography (fig. 2a) showed a dilated cystic duct and a dilated CBD, proximal to the obstructing stone in the cystic duct. Figure $2 \mathrm{~b}$ depicts a stone in the cystic duct which moved slightly with catheter use during ERCP, causing relief of obstruction in the CBD. The cystic duct stone was moved to the CBD with a biliary balloon. Then, a mechanical basket lithotripter (fig. 3a) was used to crush the stone, which was now lying in the CBD. Stone fragments were delivered out with a large extraction balloon. A dilated cystic duct and CBD were noted at the end of the procedure after complete stone extraction (fig. $3 \mathrm{~b}$ ). The patient was clinically stable after the procedure and had an uneventful recovery. He was seen 7 weeks later in the clinic. His skin and scleral icterus had resolved completely. Epigastric pain did not recur after the procedure. He is scheduled for an elective cholecystectomy.

\section{Discussion}

Mirizzi syndrome has been classified into four types. Type 1 Mirizzi syndrome refers to extrinsic compression of the CBD with intact (1A) or obliterated (1B) cystic duct without a cholecystocholedochal fistula [13]. Three other types have a cholecystocholedochal fistula and vary in terms of size, expressed as percentage of the CBD width. Type 2 includes all cholecystocholedochal fistulas under $33 \%$ of the CBD width; type 3 encompasses all cholecystocholedochal fistulas ranging from 33 to $66 \%$ of the CBD width; type 4 includes cholecystocholedochal fistulas more than $66 \%$ of the CBD width [13]. A cholecystoenteric fistula that complicates the other types of cholecystobiliary fistulas was also described [14]. Surgery is the mainstay of therapy. Diagnosis of Mirizzi syndrome prior to surgery is 
Rayapudi et al.: Mirizzi Syndrome with Endoscopic Ultrasound Image

important so that bile duct injury can be avoided [15], however preoperative diagnosis is possible only in a small percentage of patients [5]. Cui et al. [9] detailed the surgical approach depending on the variants of Mirizzi syndrome. In type 1 syndrome, cholecystectomy (open and laparoscopic) and partial cholecystectomy were effective. Intraoperative CBD exploration may be necessary to exclude other causes of obstructive jaundice. For Mirizzi syndrome types 2 and 3, partial cholecystectomy without removal of the portion of the gallbladder around the fistula margin may be attempted. In some cases, bile duct repair using the gallbladder or the cystic duct was needed. T tubes were used for biliary decompression and support. A bilioenteric anastomosis may be required depending on the size of the fistula in type 3 Mirizzi syndrome. For Mirizzi syndrome type 4, the entire wall of the CBD would be destroyed, so bilioenteric anastomosis with Roux-en-Y hepaticojejunostomy was the preferred method of surgical management. The laparoscopic approach to Mirizzi syndrome is controversial and has been recommended to be restricted to type 1 patients with careful patient selection $[9,10]$.

We reported here a case of Mirizzi syndrome with a rare EUS image. The syndrome needs to be suspected in any patient who presents with obstructive jaundice, and surgery remains the mainstay of therapy.

\section{References}

1 Lakhtakia S, Gupta R, Tandan M, et al: Mirizzi's syndrome: EUS appearance. Gastrointest Endosc 2006;63: 322-323; discussion 323.

2 Beltran MA, Csendes A, Cruces KS: The relationship of Mirizzi syndrome and cholecystoenteric fistula: validation of a modified classification. World J Surg 2008;32:2237-2243.

-3 Ibrarullah M, Saxena R, Sikora SS, Kapoor VK, Saraswat VA, Kaushik SP: Mirizzi's syndrome: identification and management strategy. Aust N Z J Surg 1993;63:802-806.

4 Becker CD, Hassler H, Terrier F: Preoperative diagnosis of the Mirizzi syndrome: limitations of sonography and computed tomography. AJR Am J Roentgenol 1984;143:591-596.

5 Zhong H, Gong JP: Mirizzi syndrome: experience in diagnosis and treatment of 25 cases. Am Surg 2012;78: 61-65.

-6 Tsuyuguchi T, Saisho H, Ishihara T, Yamaguchi T, Onuma EK: Long-term follow-up after treatment of Mirizzi syndrome by peroral cholangioscopy. Gastrointest Endosc 2000;52:639-644.

7 Binmoeller KF, Thonke F, Soehendra N: Endoscopic treatment of Mirizzi's syndrome. Gastrointest Endosc 1993;39:532-536

E England RE, Martin DF: Endoscopic management of Mirizzi's syndrome. Gut 1997;40:272-276.

-9 Cui Y, Liu Y, Li Z, Zhao E, Zhang H, Cui N: Appraisal of diagnosis and surgical approach for Mirizzi syndrome. ANZ J Surg 2012;82:708-713.

10 Kwon AH, Inui H: Preoperative diagnosis and efficacy of laparoscopic procedures in the treatment of Mirizzi syndrome. J Am Coll Surg 2007;204:409-415.

-11 Targarona EM, Andrade E, Balagué C, Ardid J, Trías M: Mirizzi's syndrome. Diagnostic and therapeutic controversies in the laparoscopic era. Surg Endosc 1997;11:842-845.

12 Haribhakti SP, Awasthi S, Pradeep R, Kapoor VK, Kaushik SP: Carcinoma gallbladder: atypical presentations and unusual associations. Trop Gastroenterol 1997;18:32-34.

13 Pelaez-Luna M, Levy MJ, Arora AS, et al: Mirizzi syndrome presenting as painless jaundice: a rare entity diagnosed by EUS. Gastrointestinal Endoscopy 2008;67:974-975; discussion 975.

14 Csendes A, Muñoz C, Alban M: Síndrome de Mirizzi - fístula colecistobiliar, una nueva clasificación. Rev Chil Cir 2007;59(suppl):63-64.

15 Hazzan D, Golijanin D, Reissman P, et al: Combined endoscopic and surgical management of Mirizzi syndrome. Surg Endosc 1999;13:618-620. 


\section{Case Reports in \\ Gastroenterology}

\begin{tabular}{l|l}
\hline Case Rep Gastroenterol 2013;7:202-207 \\
\hline DOI: 10.1159/000351170 & $\begin{array}{l}\text { ○ 2013 S. Karger AG, Basel } \\
\text { www.karger.com/crg }\end{array}$ \\
\hline
\end{tabular}

Rayapudi et al.: Mirizzi Syndrome with Endoscopic Ultrasound Image

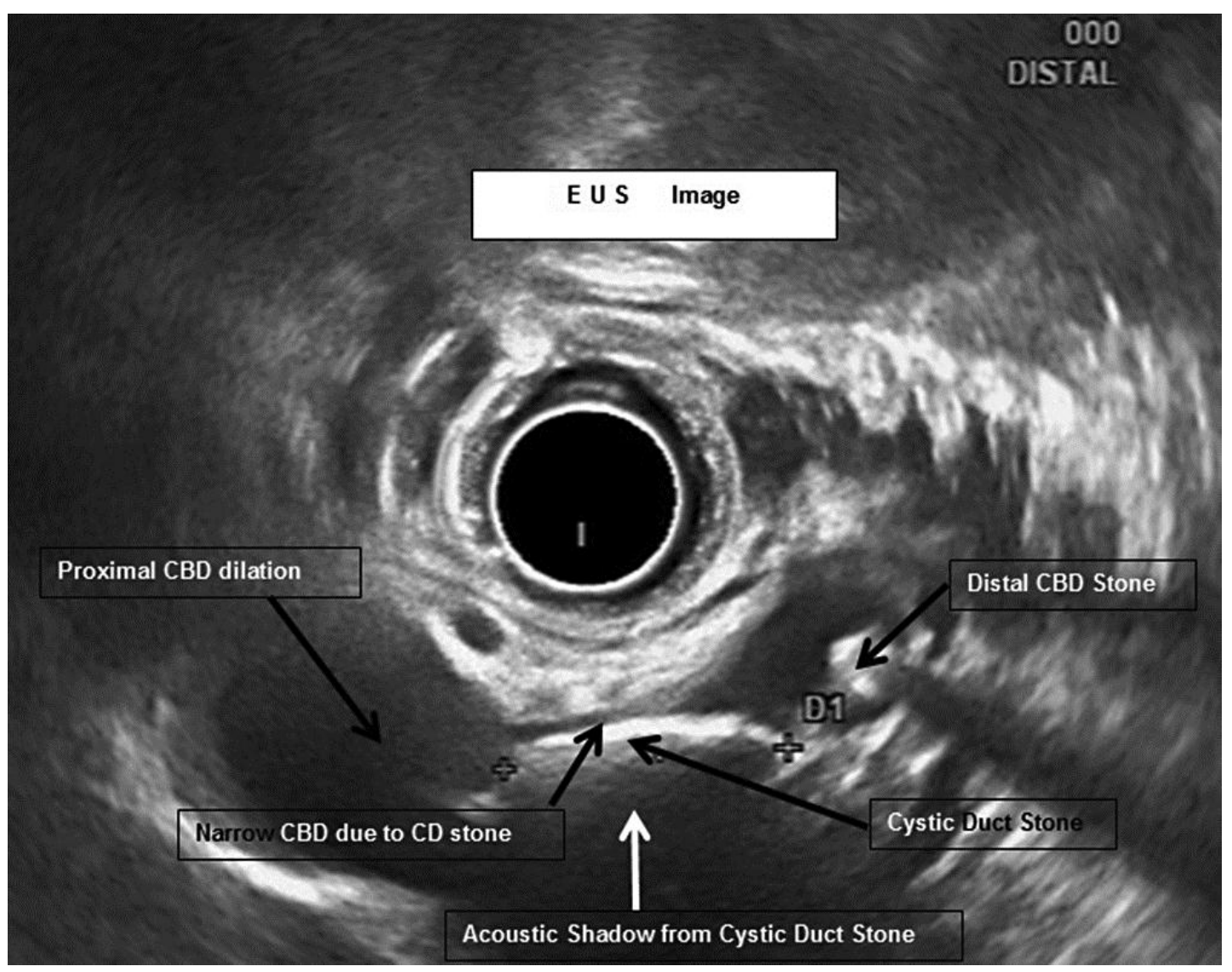

Fig. 1. EUS image showing type 1 Mirizzi syndrome.
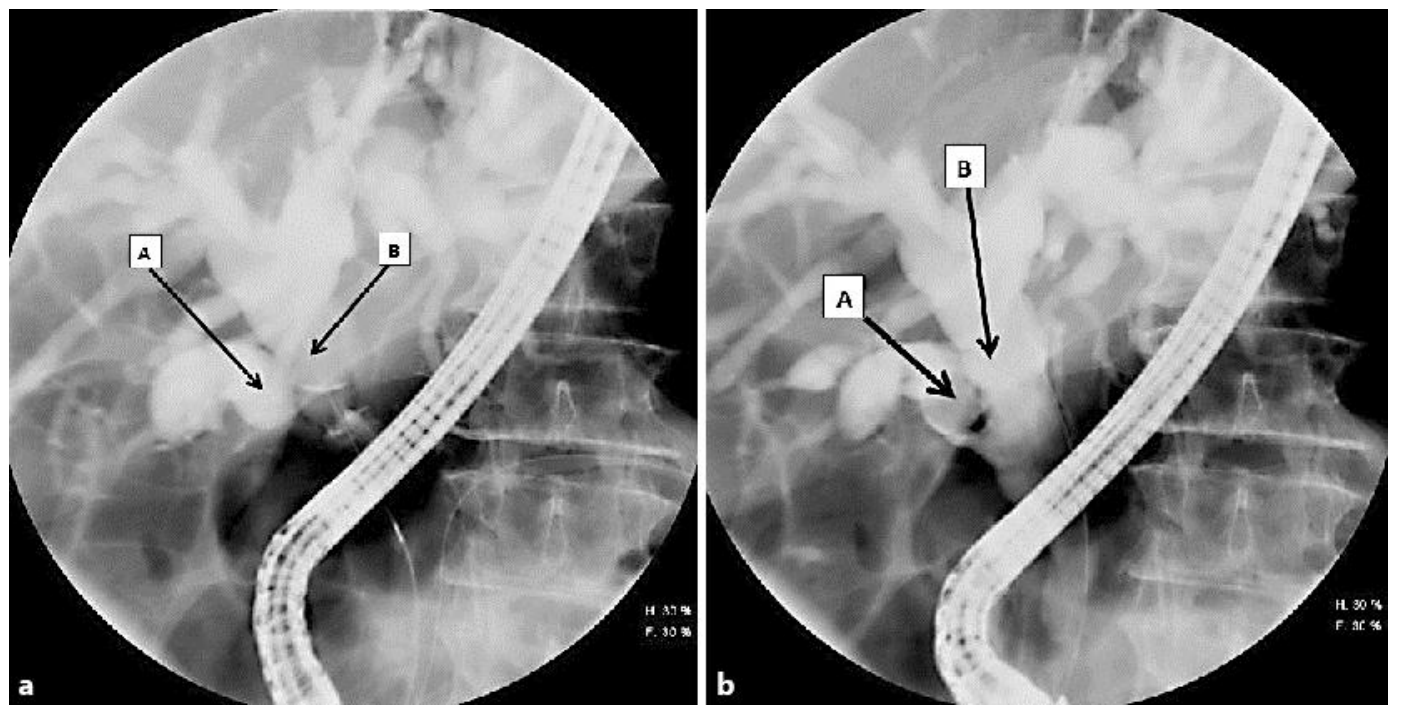

Fig. 2. a Dilated cystic duct (A) and dilated CBD (B), proximal to the obstructing stone in the cystic duct during cholangiography. $\mathbf{b}$ A stone in the cystic duct $(A)$ which moved slightly with catheter use during ERCP, causing relief of obstruction in the CBD (B). The picture shows a dilated CBD (B). 


\begin{tabular}{l|l}
\hline Case Rep Gastroenterol 2013;7:202-207 \\
\hline DOI: 10.1159/000351170 & $\begin{array}{l}\text { ○ 2013 S. Karger AG, Basel } \\
\text { www.karger.com/crg }\end{array}$ \\
\hline
\end{tabular}

Rayapudi et al.: Mirizzi Syndrome with Endoscopic Ultrasound Image

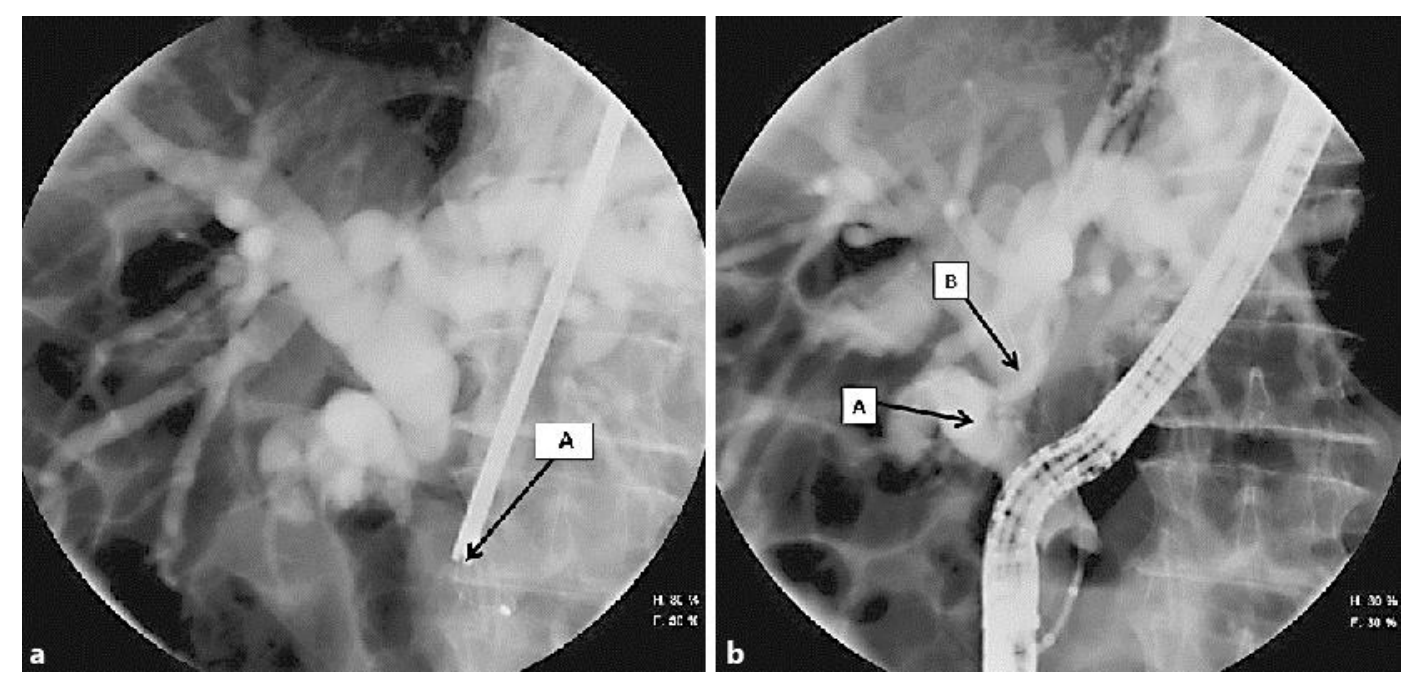

Fig. 3. a The stone being crushed with a mechanical basket lithotripter (A). b Dilated cystic duct (A) and CBD (B) after complete stone extraction. 